A. Pramesh Rao, G. Swarup and Gopal-Krishna, eds.

\title{
The Nuclear Structure of the Giant Radio Galaxy 3C236
}

\author{
W.W.Tian ${ }^{1,2}$, R.T. Schilizzi ${ }^{3,4}$ and R. $\operatorname{Nan}^{1}$ \\ ${ }^{1}$ Beijing Astronomical Observatory and Beijing Astrophysics Center of \\ National Astronomical Observatories, CAS, China \\ ${ }^{2}$ Max-Planck-Institut für Radioastronomie, Bonn, Germany \\ 3 Joint Institute for VLBI in Europe, Dwingeloo, The Netherlands \\ ${ }^{4}$ Leiden Observatory, Leiden, The Netherlands
}

\begin{abstract}
We present the nuclear structure of the radio galaxy 3C236 obtained by VLBI and MERLIN observations at both 1.663 and $5 \mathrm{GHz}$. Comparison with an HST WFPC-2 image of 3C236 suggests that the radio axis is at an angle of $\sim 30^{\circ}$ to the plane of the sky, and $3 \mathrm{C} 236$ has an overall size of $4.5 \mathrm{Mpc}$ and contains the longest jet known in the universe.
\end{abstract}

\section{Introduction}

$3 \mathrm{C} 236$ is the largest radio source known in the universe. Its total radio luminosity of $1.5 \times 10^{43} \mathrm{erg} / \mathrm{sec}$ puts it in the transition zone between FR1 and FR2 systems. Its morphology is also intermediate between FR1 and FR2 on large scales. A study of the evolution of $3 \mathrm{C} 236$ should constrain models for the evolution of radio sources in general.

\section{Results}

Figure 1 (left) shows the $2 \mathrm{kpc}$ radio structure in the nucleus of the galaxy at 1.663 GHz. There are two main emission areas, the central strong emission region (B1-B2-C) and the extended radio lobe in the northwest (A). Fig. 1 (right) shows a detailed image of the central strong emission region at $5 \mathrm{GHz}$. The centre of component B2 is revealed as a multi-component structure in position angle $112^{\circ}$, with the brightest component at the eastern end. The presence of component $\mathrm{B} 1$ is also visible in the data although it is well resolved at this resolution. This is the highest angular resolution we have on the nucleus itself, and it is remarkable that at this resolution, the structure appears to be a one-sided jet whereas, on all other scales, the structure is two-sided.

Fig. 2 (left) shows the absorption model for the galaxy derived by de Koff et al. (2000), in which the main component of dust is in the form of a ring of radius $\sim 5 \mathrm{kpc}$. From the ellipticity of the disk, we derive an apparent inclination angle of the radio source to the line of sight of $\sim 60^{\circ}$ assuming that the radio jets are approximately perpendicular to the dust ring. This implies that the NW jet is approaching, and that the true size of $3 \mathrm{C} 236$ is $4.5 \mathrm{Mpc}$ (Schilizzi et al. 2000). The Westerbork image of $3 \mathrm{C} 236$ at $327 \mathrm{MHz}$ in Fig. 2 (right) provides strong 

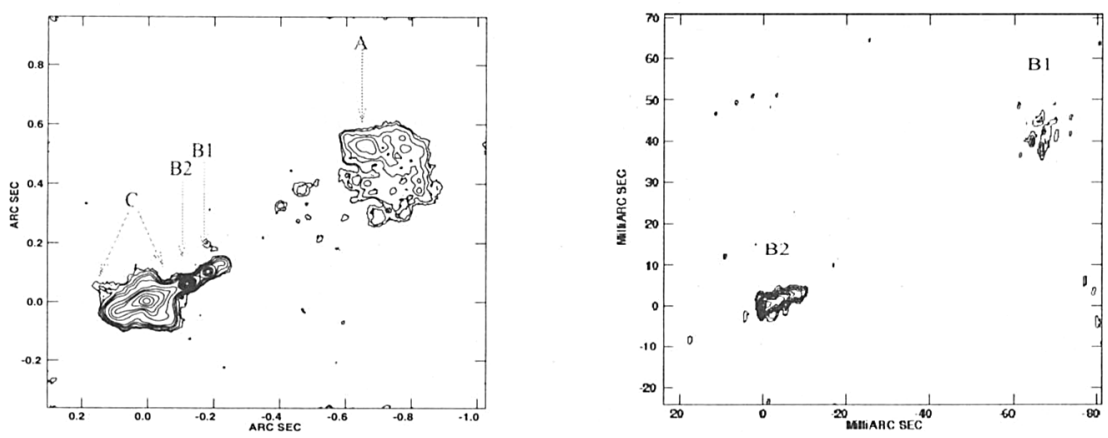

Figure 1. The central $2 \mathrm{kpc}$ in $3 \mathrm{C} 236$ at 1.66 and $5 \mathrm{GHz}$; restoring beams are 25 and 1.5 mas in diameter, respectively; contour levels are: $-1,1,1.5,2.5,3.5,5,10,15,20,30,40,50,60,75,90 \%$ of the peak brightness $251 \mathrm{mJy} /$ beam (left) and 1, 2, 3, 8, 12, 20, 40, 60, 90\% of the peak brightness $85 \mathrm{mJy} /$ beam (right)
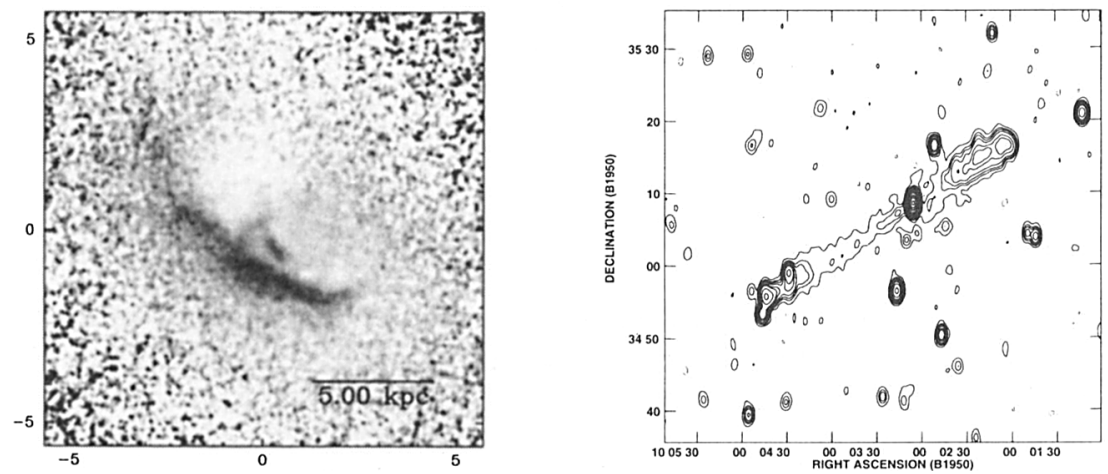

Figure 2. (left) Absorption model for 3C236 by de koff et al. (2000) from $7000 \mathrm{~A}^{0}$ HST data; (right) $327 \mathrm{MHz}$ WSRT image of $3 \mathrm{C} 236$ with a restoring beam of 1 arcmin; contour levels are $-0.1,0.1,0.3,0.5$, $0.8,1.4,2.4,8,15,30,40,60,90 \%$ of the peak brightness, which is $7.24 \mathrm{Jy} /$ beam

evidence that the jet to the SE has been on for most of the life of the radio source. At the resolution of the measurements, the jet appears to originate in the nucleus and continue for $\sim 2.5 \mathrm{Mpc}$ before terminating in a double hotspot (Barthel et al. 1985). This is the longest jet known in the universe.

\section{References}

Barthel P. D., Schilizzi R. T., Miley G. K., et al., 1985, A\&A, 148, 243

De Koff S., Best P., Baum S. A., Sparks W., et al., 2000, Ap. J., in press

Schilizzi R. T., Tian W. W., Conway J. E., Nan R., Miley G. K., Barthel P. D., Normandeau M., 2000, A\&A, in preparation 